\title{
COVID-19 Vaccine An Engineering perspective
}

\author{
Ho Teck Tuak, PhD. \\ Keywords: Vaccine, Side-Effects, Unknown
}

What is a Vaccine, or as one has understood it to be thus far? A Vaccine is something a person takes maybe at childhood stage, and it is taken once in a lifetime, for example, Chickenpox Vaccine.

Any food that is taken orally into the body, is likely to do something to the body, whether it is good or bad. There is likely no food taken into the body and does nothing to the body. There are claims that medicine taken into the body will leave the body after $24 \mathrm{hrs}$. This is likely incorrect. It must have done something inside the body before it ever leaves the system. Similarly, any medicine or vaccine taken into the body will do something to the body. As medicine is a chemical or biological compound it may do more than what it is mainly intended for and is likely to do something else and is called side-effects. Therefore, all medicine will likely cause side effects.

As food is consumed it will likely mainly affect the body's "Operational" system. For drugs, it is likely to affect the body's "Functional" system. For drugs that affect the genetics realm, it is likely to affect the body's "Basic" system. It is likely affecting the basic system will likely initiate evolution.

Therefore, drugs and vaccines need to go through thorough testing and trials to understand what it does besides the main objectives. Therefore, such drugs have to be tested and trialed for likely 10 years or more because that is likely how the body will subtly react to side effects and show it out over time. It is likely the body will compensate from other parts to the parts that lacks until it cannot compensate anymore and show it out. 10 years is likely a reasonable yardstick for a body, as diseases likely appear in that amount of time if one mistreats its body.

Therefore, the vaccine that requires booster shots, and bi-annual booster shots cannot be classified confusingly as a vaccine in the traditional sense. It is likely only classified as a Temporary-Vaccine or its equivalent. If the body forgets, it cannot be a vaccine. Not doing so is likely a cause for medically regulatory misleading advertisements. Is this a case of if the booster shots are not continued the patient is back to being unvaccinated? Therefore, continuing to take booster shots after booster shots becomes a Vaccine Addiction. It is likely good for the manufacturer selling the drugs but it cannot be a good thing or even practical for the consumer or patient. It cannot be counted as a longterm solution.

Such a Vaccine may not be the solution because real-life studies have continually shown many of both the unvaccinated and vaccinated die from the disease. If one dies from the disease, it is likely one is able to spread it to others. It is likely both happen to be at the wrong place and time to have caught the disease. It is likely both who caught the disease did not observe mask-wearing and physical distancing or other safety measures. It is likely an unvaccinated and observing mask-wearing and physical distancing and other safety measures are more likely to not catch the disease than a vaccinated and do not observe the safety measures. Blaming almost everything on the unvaccinated 
who is either by choice or medical requirement is not evidence-based and cannot be proven or sustained. Promoting taking such vaccine as an ultimate solution unknowingly is a cause for spreading a false sense of security, and leads to people behaving with a false sense of security.

A person with medical conditions restricting them from taking such vaccine and the medical professional making a decision whether to go ahead or not is only doing a guess.

In times many will develop non-COVID underlining illnesses. Is this group destined for death anyway even if they are vaccinated?

Is the unvaccinated subjecting to human rights violation and maybe even death due to being shut out of essential services because there is no arranging for equivalence and are left out dry?

As the body consumes more and more of the same drugs or vaccines, the side effects will likely grow and intensify.

What one does not want is to find out after a long period that one likely evolute and grows shorter, or run less far, or tired easily, or does undesirables to their offspring. 